\title{
Surprisingly Neuroplastic Human Brains: Reading, Science, Philosophy, Theology
}

\author{
Holmes Rolston III
}

\begin{abstract}
Human brains, dramatically more complex than anything else in the known universe, are marvelously mutable. Recent neuroscience focuses on how humans create cumulative transmissible cultures which in turn shape mental development. When cultures become literate, cognitive powers escalate. Although until recently only a comparative few learned to read and write, this takes place with the serendipitous re-use of pattern recognizing capacities, such as those for recognizing faces. With sustained reading diligence, as required during education in science, philosophy, and theology, this results in advanced cognitive skills.
\end{abstract}

\section{KEYWORDS}

Neuroplasticity; transmissible cultures; literacy; neural reuse; visual word-form re-use; exaptation; reading; writing; advanced language processing

Literate humans can display serendipitous reading skills. This recent discovery in neuroscience is of considerable philosophical interest. We here will connect accounts in the neuroscience of plasticity in human minds with increased powers of cognitive comprehension in highly educated scholars. This continues and elaborates several decades of discovering how remarkable and complex are all human minds, those of more ordinary people as well, across their span of life from infancy to senior citizens.

These new skills may, in some circumstances, contribute to the survival of the fittest. The better readers rear successfully more children, who graduate from college more often. But the arrival of these more fit does not depend on any genetic change. Rather it depends on a culturally arising neural re-use in those cultures where advanced education into reading skills is possible. The careers of both these better readers and their children are not fully "preformed" in their genetics. Having good genes is necessary but not sufficient for this outcome. An advanced form of cultural inheritance is required that opens up an environment with new linguistic visual possibility space, which must be taken advantage of by these new beneficiaries.

Desiring and cultivated these advanced reading skills, beyond any contribution to the survival of the fittest, will frequently follow an intrinsic interest in the deeper questions themselves - the search for the Higgs boson, dark energy, for divinity, the origins of the cosmos, of life, or cultural neuroplasticity. This quest may continue across decades during which child-bearing and rearing is a past stage in one's life. Or it may be present in those who have chosen a childless career, such as monks and nuns.

\section{Neuroplasticity}

First, we should recall discoveries of generic neuroplasticity, as the background against which these discoveries of cultural neural re-use must be set. Humans have hyperimmense 
brains. In the $1500 \mathrm{cc}$. of human neural networks, there is more operational organization than anywhere else on Earth, or in the universe so far as is known. Humans have lived in cultures for perhaps a million years, during which time they have reproduced across thousands of generations. There is every reason to expect that over these millennia, those humans will do best reproductively who do best culturally; and vice versa, that a genotype will be selected to produce a culturally congenial phenotype. So there is gene-culture coevolution. If, as Aristotle put it, man is a political animal (Politics I, 2, 1253a), then human nature will be adapted for social life. Humans will have a range of motivations, inclinations, propensities, emotions that equip them for living in the culture.

Apparently, the best strategy for slow-paced genes that need to succeed in more rapidly developing cultures is not to build a relatively inflexible mind whose pace and preferences are genetically biased for this or that culture, but to build flexible minds that can make preferences independently of genetic bias. The mind may need some dispositional "modules" for generic behaviors (picking mates, helping family, obeying parents, being suspicious of strangers, or dealing with non-cooperators by ostracizing them, or preferring savannah type landscapes). But equally the genes will produce teachable, open, critical minds that can evaluate cultural options for their functional usefulness and for their contribution to a meaningful life. The direction of selection in humans, as evidenced by their enormous potential for diverse cultures (from those of the Neanderthals to a high-tech computer age), all of which require intelligence in various roles, will then select for an unspecialized intellect with open educable capacity. The resulting brain is quite plastic, forging properties enabled by our genes, but shaped by our experience, environmental, and social. Newborns from one culture are regularly transferred to, reared and educated in another culture-and succeed quite well. In the vocabulary of neuroscience, we have "mutable maps" in our cortical representations, formed and re-formed by our deliberated changes in thinking and resulting behaviors.

For example, with the decision to play the violin well, and resolute practice, young string musicians alter the synaptic connections and thereby the structural configuration of their brains to facilitate fingering the strings with one arm and drawing the bow with the other. ${ }^{1}$ Likewise, musicians enhance their hearing sensitivity to tones, enlarging the relevant auditory cortex by $25 \%$ compared with non-musicians. ${ }^{2}$ Musicians attune their brains the better to deal with their tunes.

With the decision to become a taxi driver in London, and long experience driving about the city, drivers likewise alter their brain structures, devoting more space to navigationrelated skills than non-taxi drivers have. Taxi drivers drive their brains into better shape for driving around the city. "There is a capacity for local plastic change in the structure of the healthy adult human brain in response to environmental demands." 3 Similarly, researchers have found that "the structure of the human brain is altered by the experience of acquiring a second language." ${ }^{4}$ Or by learning to juggle. ${ }^{5}$

The human brain is as open as it is wired up. Our minds shape our brains. We form a synaptic self; synapses and experiential self are reciprocal processes. One can say that finding differing locations in the brain where differing kinds of mental activities takes place is evidence for the physical basis of our mental activities. This is true. But another way to interpret the same evidence is that our mental decisions to become a violin player, taxi driver, or learn a second language re-allocate brain locations to new functions in support of these decisions. Violin players, taxi drivers, jugglers use highly localized areas 
of the brain. But other skills, such as gaining a higher education, are more pervasively distributed. The authors of a leading neuroscience text use the violin players as an icon for us all, and conclude: "It is likely that this is an exaggerated version of a continuous mapping process that goes on in everyone's brain as their life experiences vary." We have little apparatus to measure such more global synaptic changes, but every reason to think they are there. ${ }^{7}$

Minds employ and reshape their brains to facilitate their chosen ideologies and lifestyles. Our ideas, ideals, and our practices configure and re-configure our own sponsoring brain structures. We neuroimage blood brain flow to find that such thoughts can re-shape the brains in which they arise. This, in turn, can affect bodily behavior. Michael Merzenich, a neuroscientist, reports his increasing appreciation of "what is the most remarkable quality of our brain: its capacity to develop and to specialize its own processing machinery, to shape its own abilities, and to enable, through hard brainwork, its own achievements."

Enlarging this flexibility in individual minds, we must also recognize that such selfdevelopment requires an appropriate and enriching cultural context.

We owe our success to our uniquely developed ability to learn from others. This capacity enables humans to gradually accumulate information across generations and develop welladapted tools, beliefs, and practices that are too complex for any single individual to invent during their lifetime. ${ }^{9}$

The key point is that, in the case of humans and of the evolutionary history of the genus Homo at least, the environment is also, importantly, the cultural environment. ... Functional rearrangements of brain circuits can be triggered by repeated external stimuli deriving from cultural practices requiring specific education and training. ${ }^{10}$

Human culture is cumulative-that is, it develops and becomes more and more complex by building on the knowledge and cultures of previous individuals and groups over generations. ... Over time cumulative culture results in cultural traits (e.g. behavior patterns, artefacts, traditions) that are more complex than one individual could have invented alone. ${ }^{11}$

"Culture provides the impetus for new tools of many different kinds-whether calculating, playing the piano, reading right to left, or speaking Arabic."12 We inherit this endowment.

Humans have a double-level orienting system: one in the genes, shared with animals in considerable part; another in the mental world of ideas, as this flowers forth from mind, for which there is really no illuminating biological analogue. Humans live under what Peter Richerson and Robert Boyd call a dual inheritance system. They find "that the existence of human culture is a deep evolutionary mystery on a par with the origins of life itself." "Human societies are spectacular anomaly in the animal world."13 The genesis of culture is as remarkable as the genesis in nature; it is nature's most remarkable genesis. The genes outdo themselves. The central idea is that acquired social knowledge and behavior is learned and transmitted from person to person, by one generation teaching another.

Humans learn what they realize others know; they employ these ideas and resulting behaviors; they evaluate, test, and modify them, and, in turn, teach what they know to others, including the next generation. Ideas pass from mind to mind, in large part through the medium of language, with such knowledge and behavior resulting in a greatly rebuilt, or cultured, environment. "Culture" is "the driving force of human cognition." "Humans live in a "cultural niche." There is a "ratchet effect," more so in cultures with writing. The human transition in such cultures is exponential, non-linear, reaching 
extraordinary epistemic powers through the inheritance of ongoing and upbuilding cultures.

Humans are born with under-developed brains (with great possibilities) and take two decades to grow up (neoteny). ${ }^{15}$ Children require many years of parental care and are long educated in a highly social, particular cultural environment. This permits both the gradual development of the brain circuits that support their cognitive capacities and their exposure to diverse challenges and possibilities within their environments as they mature. "Humans are unique in the nature of their sociality, ecological adaptations, and, most importantly, in complete reliance on culture as the extrasomatic, transgenerationally transmitted behavioral adaptation." ${ }^{16}$ Genes and culture co-evolve.

Eventually, so far as there is still adaptive selection, the variation on which selection acts does not arise in the genes, but in the mind, ideational variation not mutations in DNA. The selection, if it remains at times natural selection (more offspring in the next generation) passes over into ideational, cultural selection (Einstein over Newton, Jesus transforming Judaism). Further, as already noted, this mental, ideational searching is driven by an intrinsic interest in the deeper questions themselves.

So we know that neuroplasticity makes education possible, including higher education, provided that there is a cultural opportunity. Students taking an under-graduate college degree are re-mapping their minds, the more so if they continue with a graduate degree. Presumably too (and allowing for outlying genius), they can do this better in the better universities, which both make available the knowledge inherited from centuries of cumulative transmissible culture and offer a contemporary research-oriented and open environment.

\section{Cultural Neural Re-Use}

We advance to the idea of cultural neural re-use. Here existing brain circuitry, already serving cognitive functions is commandeered, co-opted, and re-used (at least in significant part) to serve novel cognitive functions. New brain networks are formed as one acquires a new cultural ability. The re-used region will already be networked with other regions of the cortex and, upon the novel use, these networked connections will also be re-configured. This is a novel form of flexibility, involving neural creative serendipity or exaptation.

David Anderson concludes:

It is quite common for neural circuits established for one purpose to be exapted (exploited, recycled, redeployed) during evolution or normal development, and be put to different uses, often without losing their original functions. Neural reuse theories thus differ from the usual understanding of the role of neural plasticity (which is, after all, a kind of reuse) in brain organization along the following lines: According to neural reuse, circuits can continue to acquire new uses after an initial or original function is established. ${ }^{17}$

For example, studies find that the same neural capacities used for manipulating one's fingers prove also to be useful when a child learns numbers and starts math (beginning with counting on her fingers). ${ }^{18}$

Previously learned neural networks, typically still retaining those functions, are now remodelled in part to provide neural space for new functions. There are training-induced modifications in the brain as novel skills are acquired. 
Francesco d'Errico and Ivan Colagè conclude:

Cultural exaptation refers to the reuse of previously devised cultural features for new purposes. Cultural neural reuse refers to cases in which exposure to cultural practices induces the formation, activation, and stabilization of new functional and/or structural brain networks during the individual lifespan.... Repeated cycles of cultural exaptation, development of appropriate strategies of cultural transmission, and ensuing cultural neural reuse represent the fundamental mechanism that has regulated the cultural evolution of our lineage. ${ }^{19}$

Such practices will involve information transfer through intentional storage and teaching, dedicated training, behavioral copying, often using novel instruments and tools. Language will be used for engaging in conversation, giving directives, asking questions. Printed language (with figures and illustrations) will add dramatic new powers to this cybernetic, cognitive process.

\section{Visual Word-Form Re-Use}

Humans are quite good at recognizing patterns, with obvious survival benefits. I can recognize the face of my wife among the seven billion persons on Earth. Recent studies in neuroscience indicate that readers re-use pattern recognition skills already present, well developed in facial recognition, some of which are now redeployed for written language recognition. Further, with intensive training and practice in learning to read and write, as is done by skilled scholars in fields such as literature, science, philosophy, and theology, this can be honed into advanced powers for comprehension, beyond those of ordinary readers.

The invention of writing is interpreted as a case of cultural exaptation of previous devices to record information, in use since at least the Early Later Stone Age and the beginning of the Upper Paleolithic (44,000 years before present). The measurable changes in brain structure and functioning caused by learning to read are proposed as an exemplar case of cultural neural reuse. ${ }^{20}$

"We choose to focus on the brain substrates for reading ability ... as several studies in the last two decades have gathered clear evidence for the effectiveness of such a cultural practice in shaping the brain phenotype." 21

Such re-use is thought to feature what is called the "visual word form area" (VWFA), a specific brain region of the left ventral occipito-temporal cortex used during the process of reading acquisition. ${ }^{22}$ Reading accurately and with facility requires that this area be properly functioning, as shown by both fMRI imaging ${ }^{23}$ and studies of injured patients. ${ }^{24}$

So far as we know, anything we might call reading appeared in Mesopotamia about 6000 years ago. For thousands of years, only a small fraction of the population in any culture learned to read and write. This cultural elite developed specialized abilities, enabling more advanced language processing, metaphorical thinking, reading or numeracy. "Part of human cortex is specialized for cultural domains such as reading and arithmetic, whose invention is too recent to have influenced the evolution of our species.... Cultural inventions invade evolutionarily older brain circuits and inherit many of their structural constraints." This involves

the reuse, in the course of evolution, of an ancient biological mechanism in a completely different role. In cultural learning, however, this reuse obviously does not involve any genetic reshuffling, but occurs during the life span as a result of brain plasticity. ${ }^{25}$ 
Reading is therefore a perfect case of cultural neural reuse.... It is not the outcome of genetic evolution but mainly the consequence of ontogenetic exposure to an articulate cultural strategy transmitted through teaching.... The Visual Word Form Area (VWFA) as an area for reading exploits a very specific brain region (the VWFA) evolved for other functional purposes such as object and face recognition. ${ }^{26}$

When reading, one recognizes familiar words immediately. Less familiar words, perhaps rarely seen, or longer words, which one may have to pronounce in one's mind to hear the phonology, take more time. These processes take place in different, though nearby areas of the brain. Some doubt whether this area is localized enough to be tabbed a VWFA, without denying that there is neural re-use. "Reading evokes activation in a distributed set of regions." 27

Widespread literacy is only a few centuries old, so these must be culturally acquired skills re-configuring previous uses when persons are challenged with the dynamic possibilities of reading. Interestingly, in whatever the reading and writing system, whether Chinese, Japanese, French, or English, these same neural areas are re-used in diverse writing systems, with some local variations. ${ }^{28}$ This is also true even when individuals blind from birth learn to read Braille. ${ }^{29}$

This redeployment is at times by using metaphor. For example, living a life is like taking a journey; we stretch the experience of a journey into thinking about how we live life-doing so using the same neural region for both. We think of increasing numbers by putting them in a lengthening line-reusing the same neural processes. ${ }^{30}$ But we often use the same neural region to think about concepts that are not metaphorically related, such as language and music, or the words on signs on buildings to the actual building and activities within it, or language and Braille dots.

This re-use should appear in all those whose jobs require steady reading such as accountants and elementary school teachers. That will be true, but philosophers and theologians are avid readers who will learn to read and recall stored texts more rapidly in order to formulate and evaluate arguments. Mature readers can read extremely fast, up to 200 words per minute. They can scan several pages quickly to see whether there is anything likely to be useful in their current research.

Well at least the accountants and elementary school teachers are successful. They get the books balanced and the school children learn to read and write. Those philosophers and theologians, keen readers though they may be, remain in endless arguments and never settle anything. True again, but they are addressing the deepest questions. And some things do get settled between scientists, philosophers, and theologians. There is a widespread consensus for instance that the sciences, whatever their impressive successes with facts, laws, theories, are not competent alone to address the ethical and deeper value questions they may raise. There is widespread consensus that philosophers and theologians today are no longer credible, whatever their inherited metaphysics and dogmas, until they have addressed issues that the contemporary natural and social sciences raise. There is widespread consensus that one ought to respect life, and human autonomy and dignity. We have considerable responsibility to make such increased reading opportunities available to those in presently less fortunate circumstances.

In terms of the "bridges" metaphor of the Center for Theology and the Natural Sciences, these surprising discoveries in the neurosciences could have gone either way from theology to science or from science to theology. In fact, this research started when neuroscientists noticed that some injured or diseased patients were deficient in reading 
skills. But it might have been that the philosophers or theologians said, "Hey, we are experiencing quite advanced reading skills developing over persistent diligence in reading. You neurologists should look around and see if you can find out what is going on in our brains." Maybe thoughtful theologians can yet give them some tips about what serendipitous surprises the neurologists should look for next.

\section{Notes}

1. Thomas Elbert et al., 1995. "Increased Cortical Representation of the Fingers of the Left Hand in String Players," Science 270 (13 October): 305-7.

2. Christo Pantev et al., 1998. "Increased Auditory Cortical Representation in Musicians," Nature 392 (23 April): 811-4.

3. Eleanor A. Maguire et al., 2000. "Navigation-Related Structural Change in the Hippocampi of Taxi Drivers," Proceedings of the National Academy of Sciences of the United States of America 97, no. 8: 4398-403.

4. Andrea Mechelli et al., 2004. "Structural Plasticity in the Bilingual Brain," Nature 431 (14 October): 757.

5. Bogdan Draganski, 2004. "Changes in Grey Matter Induced by Training." Nature 427 (22 January): 311-2.

6. Mark F. Bear, Barry W. Connors, and Michael A. Paradiso, 2001. Neuroscience: Exploring the Brain, 2nd ed. (Baltimore: Lippincott Williams and Wilkins), 418.

7. Joseph LeDoux, 2002. Synaptic Self: How Our Brains Become Who We Are (New York: Viking).

8. Michael Merzenich, 2001. "The Power of Mutable Maps," box essay, p. 418 in Mark F. Bear, Barry W. Connors, and Michael A. Paradiso, 2001. Neuroscience: Exploring the Brain, 2nd ed. (Baltimore: Lippincott Williams and Wilkins).

9. Robert Boyd, Peter J. Richerson, and Joseph Henrich, 2011. "The Cultural Niche: Why Social Learning Is Essential for Human Adaptation," Proceedings of the National Academy of Sciences USA 108 (Supplement 2): 10918-25, on p. 10918.

10. Paolo D'Ambrosio and Ivan Colagè, 2017. “Extending Epigenesis: From Phenotypic Plasticity to the Bio-Cultural Feedback," Biology and Philosophy 32 (no. 5): 705-28, on p. 705, p. 720.

11. Emily R.R. Burdett, Lewis G. Dean, and Samuel Ronfard, 2017. "A Diverse and Flexible Teaching Toolkit Facilitates the Human Capacity for Cumulative Culture," Review of Philosophy and Psychology, p. 1, https://link.springer.com/content/pdf/10.1007\%2Fs13164-0170345-4.pdf; https://doi.org/10.1007/s13164-017-0345-4.

12. Nicholas Evans and Stephen C. Levinson, 2009. "The Myth of Language Universals: Language Diversity and Its Importance for Cognitive Science," Behavioral and Brain Sciences 32: 429-92, on p. 447.

13. Peter J. Richerson and Robert Boyd, 2005. Not by Genes Alone: How Culture Transformed Human Evolution (Chicago: University of Chicago Press), on p. 126. p. 195.

14. Ivan Colagè and Francesco d'Errico, 2018. "Culture: The Driving Force of Human Cognition," Topics in Cognitive Science, July, https://doi.org/10.1111/tops.12372.

15. Mehmet Somel, Lin Tang, and Philipp Khaitovich, 2012, "The Role of Neoteny in Human Evolution: From Genes to the Phenotype," in Hirohisa Hirai, Hiroo Imai, Yasuhiro Go, eds., Post-Genome Biology of Primates. Primatology Monographs. Tokyo, Japan: Springer.

16. Branka Hrvoj-Mihic, Thibault Bienvenu, Lisa Stefanacci, Alysson R. Muotri, and Katerina Semendeferi, 2013. "Evolution, Development, and Plasticity of the Human Brain: From Molecules to Bones," Frontiers in Human Neuroscience 7 (October, Article 707): 1-20.

17. Michael L. Anderson, 2010. "Neural Reuse: A Fundamental Organization Principle of the Brain," Behavioral and Brain Sciences 33: 245-313, on p. 245.

18. Michael L. Anderson and Marcie Penner-Wilger, 2013. "Neural Reuse in the Evolution and Development of the Brain: Evidence for Developmental Homology?" Developmental Psychobiology 55: 42-51. Also a dozen references there. 
19. Francesco d'Errico and Ivan Colagè, 2018. "Cultural Exaptation and Cultural Neural Reuse: A Mechanism for the Emergence of Modern Culture and Behavior," Biological Theory 13 (no. 4, December): 213-27, on p. 213, https://doi.org/10.1007/s13752-018-0306-x.

20. Francesco d'Errico and Ivan Colagè, 2018, on p. 213.

21. Paolo D'Ambrosio and Ivan Colagè, 2017, p. 707.

22. Stanislas Dehaene, Laurent Cohen, José Morais, and Régine Kolinsky, 2015. "Illiterate to Literate: Behavioral and Cerebral Changes Induced by Reading Acquisition," Nature Reviews Neuroscience 16: 234-44.

23. Gaël Jobard, Fabrice Crivello, and Nathalie Tzourio-Mazoyer, 2003. "Evaluation of the Dual Route Theory of Reading: A Metanalysis of 35 Neuroimaging Studies," NeuroImage 20: 693712; Fabien Vinckier, Stanislas Dehaene, Antoinette Jobert, Jean Philippe Dubus, Mariano Sigman, and Laurent Cohen, 2007. "Hierarchical Coding of Letter Strings in the Ventral Stream: Dissecting the Inner Organization of the Visual Word-Form System," Neuron 55: 143-56.

24. Laurent Cohen, Stanislas Dehaene, Lionel Naccache, Stéphane Lehéricy, Ghislaine DehaeneLambertz, Marie-Anne Hénaff, and François Michel, 2000. "The Visual Word Form Area. Spatial and Temporal Characterization of an Initial Stage of Reading in Normal Subjects and Posterior Split-Brain Patients,” Brain 123: 291-307; Raphaël Gaillard, Lionel Naccache, Philippe Pinel, Stéphane Clémenceau et al., 2006. "Direct Intracranial, fMRI, and Lesion Evidence for the Causal Role of Left Inferotemporal Cortex in Reading," Neuron 50: 191-204.

25. Stanislas Dehaene and Laurent Cohen, 2007. "Cultural Recycling of Cortical Maps," Neuron 56: 384-98, on p. 384. See also Anderson 2010.

26. Francesco d'Errico and Ivan Colagè, 2018, p. 221; Ivan Colagè, 2017. "Theology Looking at Culture Through the Lenses of Science," pages 137-55 in Michael Fuller, Dirk Evers, Anne L. C. Runehov, Knut-Willy Sæther eds., Issues in Science and Theology: Are We Special?: Human Uniqueness in Science and Theology, European Society for the Study of Science and Theology, European Conference on Science and Religion (2016, Warsaw, Poland), Cham, Switzerland : Springer Verlag, 2017; Ivan Colagè, 2015. "The Human Brain Shaping and Transcending Itself: Written Language, Brain, and Culture," Zygon 50: 1002-21.

27. Cathy J. Price and Joseph T. Devlin, 2003. "The Myth of the Visual Word Form Area," NeuroImage 19: 473-81.

28. Donald J. Bolger, Charles A. Perfetti, and Walter Schneider, 2005. "Cross-cultural Effect on the Brain Revisited: Universal Structures Plus Writing System Variation," Human Brain Mapping 25: 92-104; Marcin Szwed, Emilie Qiao, Antoinette Jobert, Stanislas Dehaene, and Laurent Cohen, 2014. "Effects of Literacy in Early Visual and Occipitotemporal Areas of Chinese and French Readers," Journal of Cognitive Neuroscience 26: 459-75.

29. Lior Reich, Marcin Szwed, Laurent Cohen, and Amir Amedi, 2011, "A Ventral Visual Stream Reading Center Independent of Visual Experience,” Current Biology 21: 363-8.

30. Michael L. Anderson, 2014. After Phrenology: Neural Reuse and the Interactive Brain (Cambridge, MA: The MIT Press), on pages 18-9, p. 22.

\section{Disclosure statement}

No potential conflict of interest was reported by the author.

\section{Notes on contributor}

Holmes Rolston III, is University Distinguished Professor and Professor of Philosophy Emeritus at Colorado State University. Some of his books are: Three Big Bangs: Matter-Energy, Life, and Mind; Genes, Genesis and God; Science and Religion: A Critical Survey; Philosophy Gone Wild; and Environmental Ethics. Rolston was laureate for the 2003 Templeton Prize in Religion, gave the Gifford Lectures, University of Edinburgh, 1997-1998, and has lectured on seven continents. A Rolston Endowed Chair in Science and Religion has been established at his alma mater, Davidson College. 